\title{
In vivo amyloid-like fibrils produced under stress
}

5 Natália A. Fontana ${ }^{1}$, Ariane D. Rosse ${ }^{1}$, Anthony Watts ${ }^{2}$, Paulo S. R. Coelho ${ }^{3}$, Antonio J. Costa6 Filho $^{1, *}$

7 1- Departamento de Física, Faculdade de Filosofia, Ciências e Letras de Ribeirão Preto,

8 Universidade de São Paulo, Ribeirão Preto, SP, Brazil

9 2- Department of Biochemistry, University of Oxford, Oxford, UK

10 3- Departamento de Biologia Celular e Molecular e Bioagentes Patogênicos, Faculdade de

11 Medicina de Ribeirão Preto, Universidade de São Paulo, Ribeirão Preto, SP, Brazil.

13 *Corresponding author: Departamento de Física, Faculdade de Filosofia, Ciências e Letras de 14 Ribeirão Preto, Universidade de São Paulo, Ribeirão Preto, SP, 14040-901, Brazil. Email: 15 ajcosta@usp.br

18 Keywords: in vivo fibrillation; fluorescence lifetime imaging; Golgi Reassembly and Stacking 


\section{Abstract}

2 The participation of amyloids in neurodegenerative diseases and functional processes has

3 triggered the quest for methods allowing their direct detection in vivo. Despite the plethora of

4 data, those methods are still lacking. The autofluorescence from the extended $\beta$-sheets of

5 amyloids is here used to follow fibrillation of S. cerevisiae Golgi Reassembly and Stacking

6 Protein (Grh1). Grh1 has been implicated in starvation-triggered unconventional protein secretion

7 (UPS), and here its participation also in heat shock response (HSR) is suggested. Fluorescence

8 Lifetime Imaging (FLIM) is used to detect fibril autofluorescence in cells (E. coli and yeast)

9 under stress (starvation and higher temperature). The formation of Grh1 large complexes under

10 stress is further supported by size exclusion chromatography and ultracentrifugation. The data

11 show for the first time in vivo detection of amyloids without the use of extrinsic probes as well as

12 bring new perspectives on the participation of Grh1 in UPS and HSR. 
1

\section{Introduction}

Amyloid fibrils have been a subject of significant interest over the years due to their pivotal participation in several neurodegenerative diseases ${ }^{[1]}$ and, more recently, functional processes. ${ }^{[2]}$ Although the structural 3D arrangement of the fibrils and factors governing their formation have been thoroughly investigated, ${ }^{[3], 4]}$ detecting amyloid-like fibrils in vivo is still not trivial. Aggregation has been monitored inside cells using GFP-labelled proteins combined with fluorescence methods. ${ }^{[5]}$ The visualization of fibrils has been achieved using strategies based on dyes that bind to amyloid plaques in excised brain tissue, ${ }^{[6]}$ and in vivo diagnostics of neurodegenerative diseases have been reported using Magnetic Resonance Imaging. ${ }^{[7]}$ To directly observe fibrils inside cells, other biochemical and biophysical approaches are still necessary.

Recently, Pinotsi et al. showed that the formation of amyloid fibrils by tau protein and lysozyme exhibited a characteristic fluorescence in the visible range. ${ }^{[8]}$ The origin of such autofluorescence was attributed to the absorption/emission of electrons delocalized after the formation of hydrogen bonds in the typical $\beta$-sheet structure of amyloids, thus allowing lowenergy electronic transitions to occur. ${ }^{[8], 9]}$ The specific molecular origin of the phenomenon is, however, still not completely understood. ${ }^{[9]}$ Despite this uncertainty, the observed autofluorescence has been firmly correlated with the amyloid formation in several cases, such as Amyloid- $\beta^{[10]}$ and $\alpha$-synuclein. ${ }^{[11]}$ Nonetheless, fibril formation monitoring was restricted to the in vitro assembly ${ }^{[10]}$ or in vivo detection using FRET between an extrinsic probe attached to the protein and the fibril. ${ }^{[11]}$

The use of autofluorescence for direct detection of in vivo fibrillation without using an extrinsic probe has not been satisfactorily explored. One reason could be the apparent lack of specificity in detecting the fibril signal due to the competing autofluorescence from the cells. Here, we report results on the in vivo formation of amyloid-like fibrils by one member of the Golgi Reassembly and Stacking Protein (GRASP) family without using an extrinsic dye for protein tagging.

GRASPs were initially implicated as participants in the structural organization of the Golgi apparatus, ${ }^{[12]}$ a central organelle in the conventional endoplasmic reticulum (ER)-to-Golgi 
1 pathway of protein secretion. ${ }^{[13]}$ However, proteins can reach the plasma membrane and leave the cell via other mechanisms. Unconventional Protein Secretion (UPS) comprises alternatives

3 through which (1) leaderless proteins (lacking the signal sequence for ER localization) are

4 secreted and (2) proteins that use the conventional secretory pathway take a different route

5 traversing from the ER straight to the plasma membrane. ${ }^{[14],[15]}$ Different types of UPS routes

6 have been reported, each dealing with different types of stress. ${ }^{[14],[15]}$ Among the four types of

7 UPS reported thus far, Types III and IV share the common participation of GRASPs. ${ }^{[16][18]}$ In particular, Type III UPS is characterized by the formation of a new GRASP-rich organelle, named Compartment for Unconventional Protein Secretion (CUPS), ${ }^{[19]}$ which leads the secretory cargo to the plasma membrane, where vesicle fuses, releasing its content.

Our group has been exploring the biophysics of GRASPs in the last few years. ${ }^{[20,21]}$ In one of our previous contributions, ${ }^{[21]}$ we described novel structural features of Grh1, the GRASP from Saccharomyces cerevisiae. We have demonstrated that Grh1 contains regions of intrinsic disorder, which seems to be a common feature among GRASPs. Furthermore, it was shown that Grh1 formed amyloid-like fibrils in vitro, and the fibrillation was independent of its C-terminal domain. ${ }^{[21]}$ In vitro fibrillation has also been observed for both human GRASPs and seems to be another general feature within the GRASP family. ${ }^{[22],[23]}$ A comprehensive review of the biophysics of GRASPs has been recently published. ${ }^{[24]}$

The capacity of Grh1 to form amyloid fibrils is still of unclear biological significance. The presence of functional amyloid aggregates in yeast has been reported previously. ${ }^{[2]} \mathrm{We}$ hypothesized that the amyloid-like form of Grh1 also occurs in vivo, and the ensemble formed is closely related to the function of Grh1 in UPS, particularly in Type III during starvation, ${ }^{[25]}$ as well as upon increase in temperature (i.e., in Heat Shock Response - HSR). ${ }^{[26]}$ In the case of starvation, intracellular $\mathrm{pH}$ drops and becomes acidic, ${ }^{[27]}$ a condition that has been seen to trigger fibrillation in vitro. As for the temperature, we have recently reported in vitro fibrillation of Grh1 at temperatures greater than $37^{\circ} \mathrm{C} .{ }^{[21]}$ In the case of yeast, the optimal temperature for its growth is $30^{\circ} \mathrm{C} .{ }^{[26]}$ The cell can handle mild temperature increases (for instance, from 37 to $41^{\circ} \mathrm{C}$ ) by

28 using the HSR. This is a coordinated event that arrests cell growth through the aggregation of 
1 Granules (SG), which are disassembled by Hsp when thermal stress ceases, and the cell returns to

2 normal growth. ${ }^{[26]}$

Here, we address one issue, namely Grh1 fibrillation in vivo, whose contribution is two-

4 fold. On the one hand, the novel direct detection of amyloid fibril formation in vivo. On the other

5 hand, the demonstration of the Grh1 fibrillation inside the cell raises new insights towards better

6 understanding fundamental aspects of UPS and HSR in yeast.

\section{Results}

\section{Grh1 forms in vitro amyloid fibrils under different conditions}

The formation of amyloid fibrils in vitro has been demonstrated for Grh1. ${ }^{[21]}$ In that study, it was shown that Grh1 fibrillates when submitted either to temperatures higher than $37{ }^{\circ} \mathrm{C}$ or changes in the dielectric constant of the medium. ${ }^{[21]}$ Those were two parameters intended to mimic the Grh1 environment (or changes to it) in the cell, i.e., heat shock response and the presence of the membrane field.

Here we complement that study by demonstrating that Grh1 also fibrillates when in acidic $\mathrm{pH}(<5.5)$, a condition that has been shown to happen during starvation. We followed a protocol similar to the one previously used ${ }^{[8]}$ based on the autofluorescence of the fibrils detected using FLIM. The fluorescence decay times were also measured and used to distinguish changes in the fibril formation. Figure S1 shows that Grh1 indeed forms fibrils in the tested conditions. Moreover, the structures formed upon heating or in acidic $\mathrm{pH}$ have different fluorescence time decays (the decay is slower in acidic $\mathrm{pH}$ ), suggesting differences in the fibril structure or the microenvironment where the fibrils are formed. Therefore, the $\mathrm{pH}$-induced in vitro fibrillation reinforces our hypothesis of fibrillation in starving conditions in yeast.

\section{Grh1 is capable of fibrillating in a cellular environment}


One of the main challenges in monitoring the fibrillation of a specific protein inside the

2 cell is to find a suitable experimental method to detect fibril formation under the conditions of

3 interest. Fluorescence Lifetime Imaging (FLIM) is based on measuring the time decay of the

4 fluorescence signal after excitation at a determined wavelength and was the method utilized to

5 detect the autofluorescence of fibrils in previous reports. ${ }^{[8-11]}$

In our case, after showing that Grh1 can form amyloid fibrils in vitro under conditions

7 that correspond to stress scenarios in the cell, the natural step after that was to ask whether Grh1

8 could also form fibrils within the cell. Hence, following the same expression protocol described

9 elsewhere, ${ }^{[21]}$ we initially turned to the heterologous expression of Grh1 in E. coli to place Grh1

10 in a cellular environment. Here, the idea was to perform experiments as if we had exchanged the

11 in vitro buffer used before for the E. coli cytoplasm.

Figure 1 shows the FLIM results obtained from E. coli cells at room temperature (Figure

$131 \mathrm{~A}$ ), at $37^{\circ} \mathrm{C}$ (Figures $1 \mathrm{~B}-\mathrm{C}$ ), and $\mathrm{pH} 4.6$ (Figure 1D). Using $20 \mathrm{mM}$ sodium acetate in the LB

14 medium as described by Wilks and Slonczewski, ${ }^{[28]}$ we prevented the bacteria from regulating its

15 internal $\mathrm{pH}$, thus turning a decrease in $\mathrm{LB} \mathrm{pH}$ value into a reduction of the $\mathrm{pH}$ of the cytoplasm.

16 The green background in the images is due to the medium autofluorescence. Rod-like structures

17 (colored in green and blue in Figure 1, where colors refer to lifetime values) resolved from the

18 background autofluorescence and likely from Grh1 appeared in the cell cultures containing Grh1

19 heated at $37^{\circ} \mathrm{C}$ or in acidic $\mathrm{pH}$ (Figures 1C-D). We discarded random fibrillation due to protein

20 overexpression by monitoring a negative control (Figure 1B), where the same E. coli strain

21 overexpressing the non-amyloidogenic protein acyl-CoA binding protein (ACBP) did not show

22 significant autofluorescence above the background signal. Other controls in acidic $\mathrm{pH}$ did not

23 show any signal either. Therefore, in the conditions that trigger in vitro fibrillation (increased

24 temperature or acidic $\mathrm{pH})$, Grh1 also fibrillated inside E. coli. 
(A)

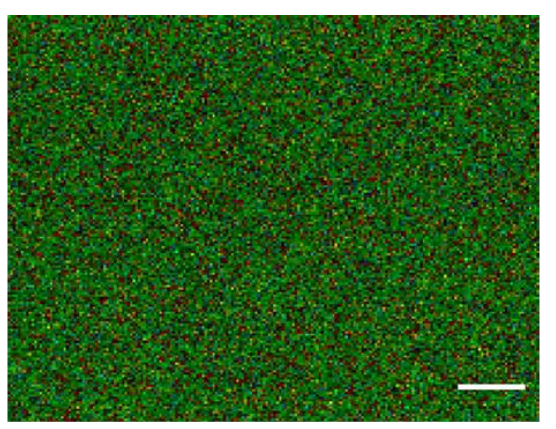

(C)

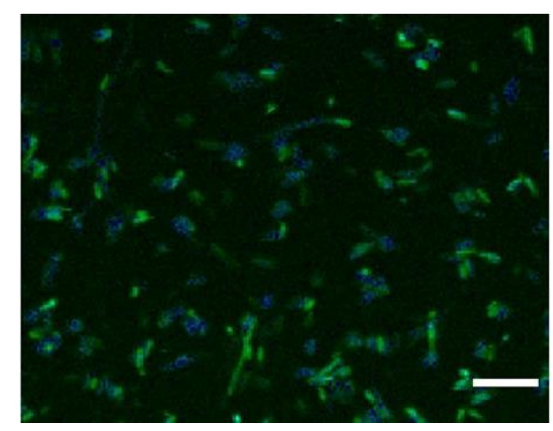

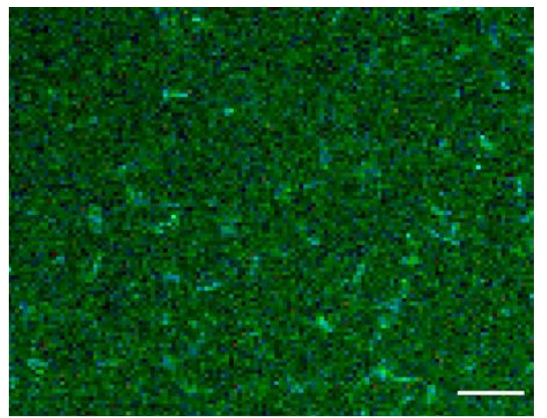

(B)

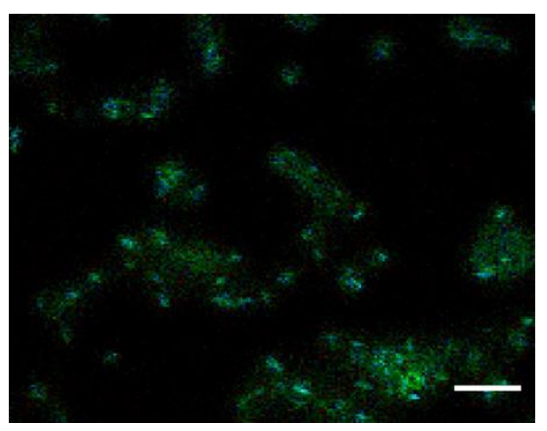

(D)

2 Figure 1: FLIM images of E. coli cells excited at $375 \mathrm{~nm}$. The images show E. coli cells expressing: (A) Grh1 at room temperature. (B) ACBP in cells heated at $37^{\circ} \mathrm{C}$ after protein expression. (C) $\mathrm{Grh} 1$ in cells heated at $37^{\circ} \mathrm{C}$ after protein expression. (D) Grh1 in cells in an acidic medium (pH 4.6). Scale bar: $10 \mu \mathrm{m}$.

Fibrillation in yeast under stress

In cell Grh1 fibrillation was also investigated in its native environment in S. cerevisiae again via FLIM experiments. It is suggested that fibrillation of Grh1 is related to stress, and therefore conditions previously described to trigger Type III UPS ${ }^{[19]}$ and HSR in yeast cells ${ }^{[27]}$ were tested. Figure 2 shows images obtained from a yeast strain called Y270, which does not carry any mutations in grhl or related genes and is hereafter referred to as Wild-type (WT) yeast. As expected, there is no autofluorescence in the control cells (Figure 2C). Fluorescence is only observed when the cells are either heated at $37^{\circ} \mathrm{C}$ (Figure 2A) or submitted to starvation (Figure 2B), conditions known to trigger HSR or Type III UPS, ${ }^{[19,27]}$ respectively.

The time decays of the fluorescence in yeast under starvation or heated at $37^{\circ} \mathrm{C}$ were

17 measured and compared with those from E. coli overexpressing Grh1 and in vitro experiments 18 (Figure 2D). The differences between in cell and in vitro time decays of fluorescence are 
1 expected since lifetime depends on the fluorophore environment. ${ }^{[29]}$ In Figure 2D, we can see that 2 each type of stress yielded different time decays, which suggests differences in the environment 3 of the fibrils upon temperature increase, starvation, or acidic $\mathrm{pH}$. This is an interesting finding 4 since variations in the cell responses to those stress conditions are expected. ${ }^{[19],[26]}$ Moreover, the 5 temperature stress in both cell types (E. coli and yeast) led to identical time decays, which were 6 somewhat longer than the time decays observed in the other two stress conditions.

(A)
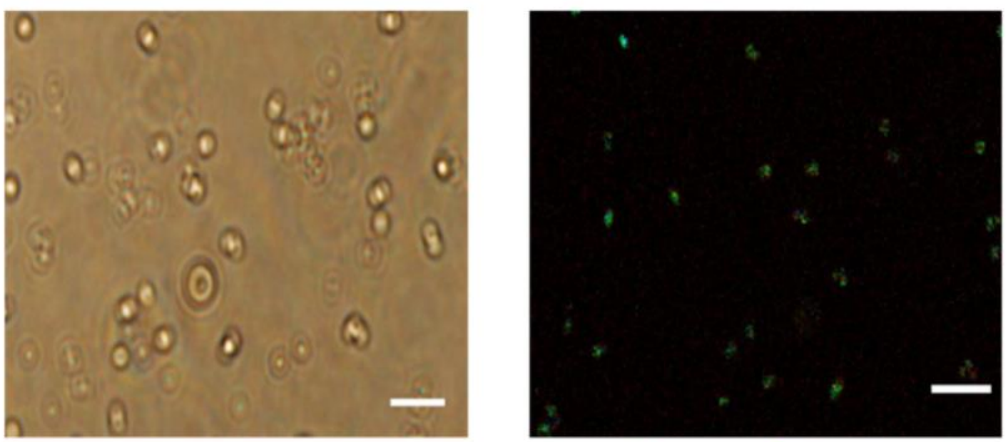

(B)
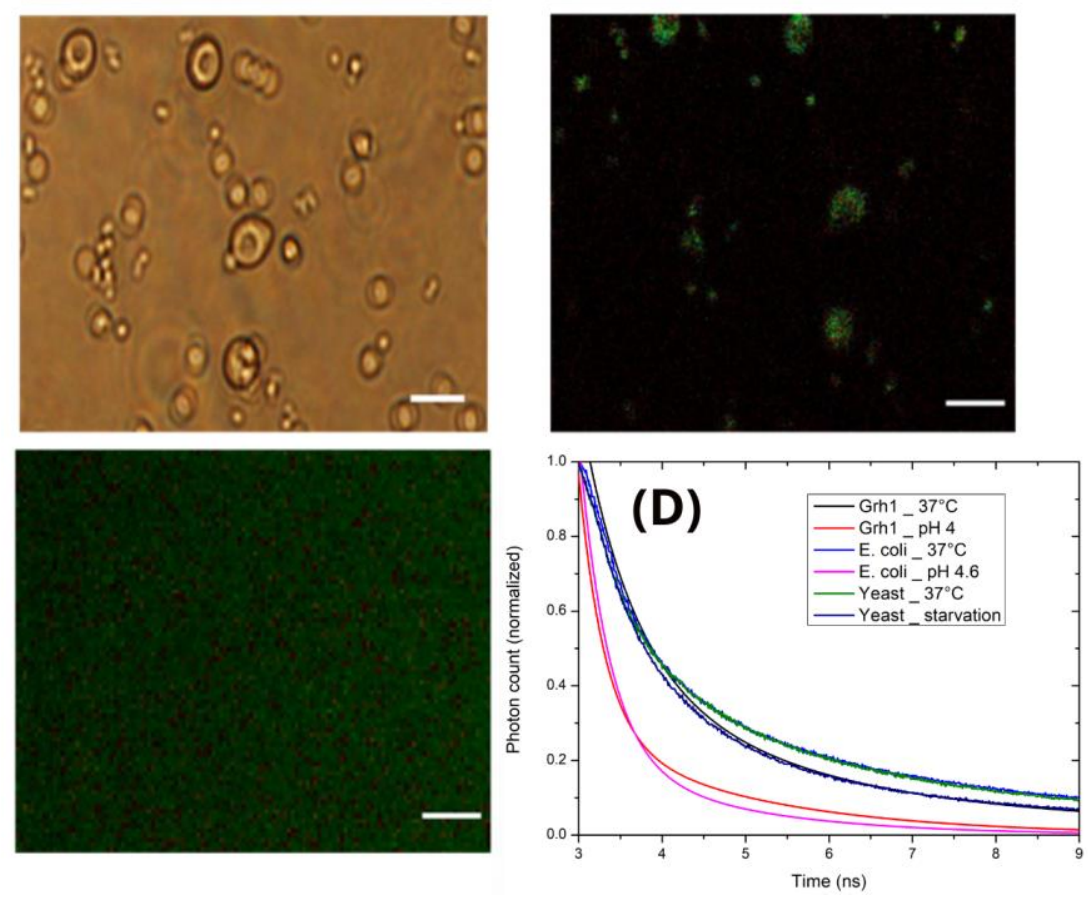

8 Figure 2: Yeast cell images in the following conditions: (A) heated to $37^{\circ} \mathrm{C}$, and (B) under 9 starvation for at least 30 minutes (left: bright field image; right: FLIM image). (C) FLIM image 10 of the control sample. (D) Time decays of fluorescence for purified $\mathrm{Grh} 1$ heated at $37^{\circ} \mathrm{C}$ (black), 11 purified Grh1 in pH 4 (red), E. coli at $37^{\circ} \mathrm{C}$ (light blue), E. coli in pH 4.6 (pink), WT yeast at $12 \quad 37^{\circ} \mathrm{C}$ (green) and starved WT yeast (dark blue). Scale bar: $10 \mu \mathrm{m}$. 
The results shown in Figures 1 and 2 indicate in cell fibrillation of Grh1. Still, it could be

4 argued that the fluorescence exhibited was due to other fibrillation processes taking place inside

5 the yeast. Similar experiments were carried out to better understand the origin of the observed

6 fibrillation process, but this time using a Grh1 knockout yeast lineage.

As expected, under physiological conditions, no autofluorescence was observed (data not

8 shown). However, in the sample heated at $37^{\circ} \mathrm{C}$ (Figure $3 \mathrm{~A}$ ), there was a change in the

9 fluorescence pattern. Not only was the FLIM signal from Grh1 lost, but also the cells appeared as

10 black dots (Figure 3A - highlighted by the red circles in the right panel). Although it was not

11 possible to quantitatively determine the loss of signal from this experiment only, the events

12 occurring in the cell suggest considerable changes took place during HSR when Grh1 was not

13 present.

In starvation, some autofluorescence in the Grh1-knockout cells (Figure 3B) was observed. To investigate this somewhat unexpected result, we also examined the pattern seen in the lifetime values by looking at the distribution of those time decays obtained from WT and

17 Grh1-knockout cells (Figure S2). A shift to longer lifetime values was observed in the 18 distribution from the Grh1-knockout cells compared with the WT sample. The lifetime corresponding to the peak of the distribution for Grh1-knockout cells increased, and the highest value of the observed lifetimes was longer (around 7.5 ns for WT cells compared to ca. $10 \mathrm{~ns}$ for

21 knockout cells). The decay times of fluorescence in both samples (Figure 3C) were also different

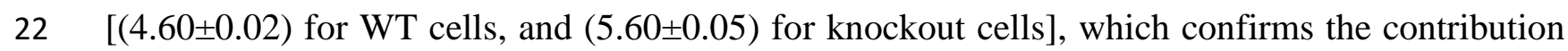

23 (or the absence of it) of Grh1 to the detected signal. An explanation for those results would be 24 that the presence of Grh1 in starvation conditions would give rise to a dominant fibrillation 25 process, thus sequestering the FLIM signal and leading to a more Gaussian-like shape of the 26 respective lifetime distribution. 
1 conditions (control) or starved for 2 hours. Once control and test group cells were grown, they 2 were lysed, and an immunoprecipitation protocol followed as described in the Material and 3 Methods. The FLIM experiments were then performed as before, but instead of whole cells, the 4 samples, in this case, consisted of trapped GFP (and, consequently, Grh1). The results for the 5 control and starvation conditions can be seen in Figure S3. While there was no signal coming 6 from the control sample (Figure S3A), in the immunoprecipitated sample from starved cells 7 (Figure S3B), the signal presented a decay of $(4.8 \pm 0.1)$ ns that is very similar to the $(4.60 \pm 0.02)$

8 ns lifetime obtained from the whole cells in starved conditions (Figure S3C). The slightly slower 9 decay might be explained by the reorganization of the fibrils once released from the cell's 10 interior. 
(A)
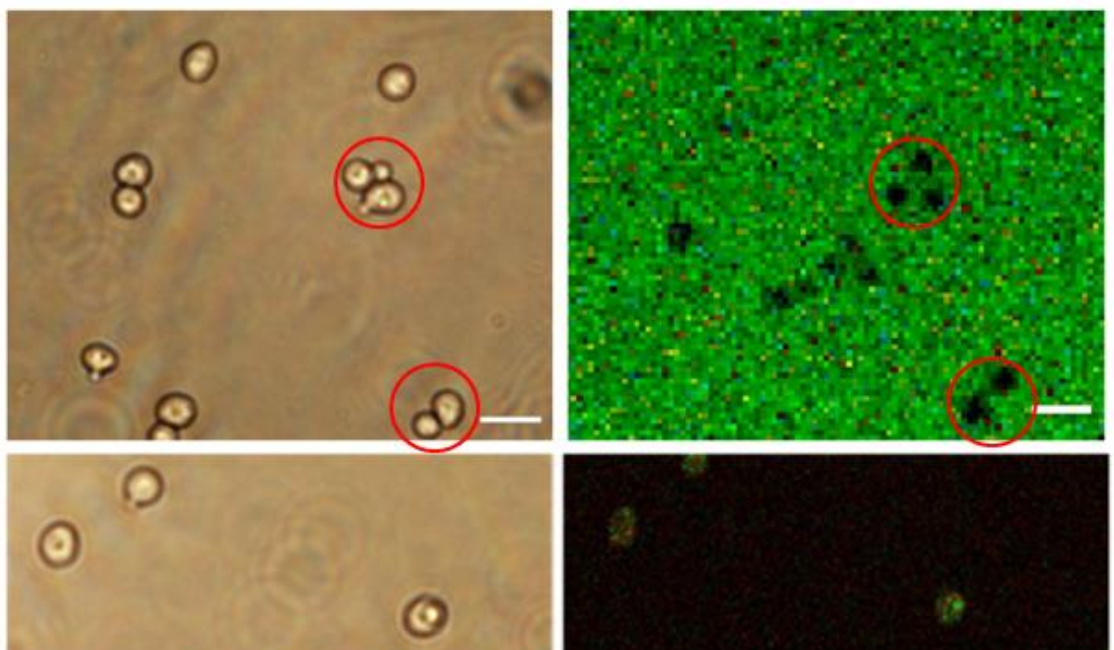

(B)
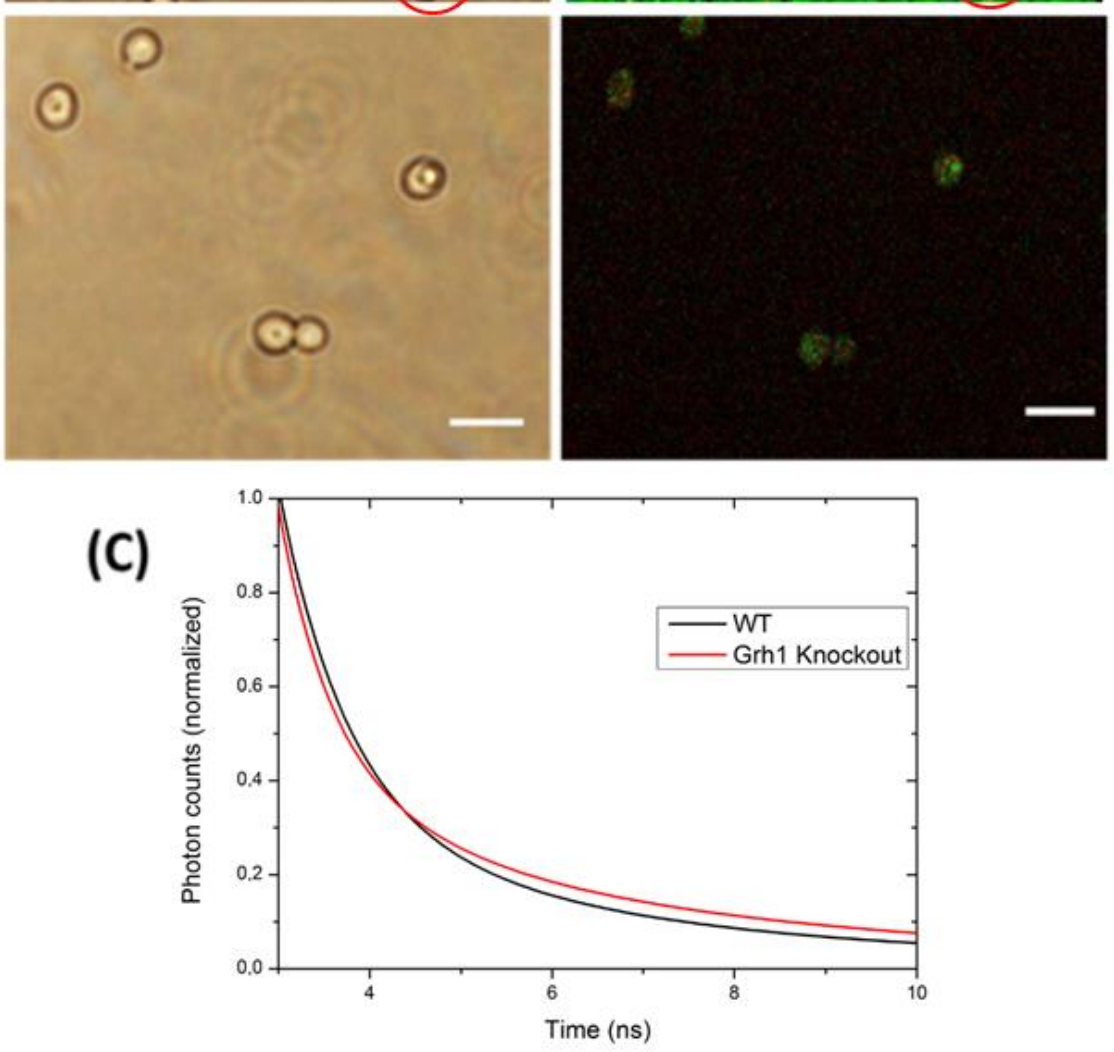

2 Figure 3: Images of Grh1 knockout yeast cells in the following conditions: (A) heated at $37^{\circ} \mathrm{C}$ 3 and (B) under starvation for at least 30 minutes (left: bright field image; right: FLIM image). (C) 4 Time decays of fluorescence for WT (black) and Grh1 knockout (red) cells. Scale bar: $10 \mu \mathrm{m}$.

5

6 
A different strategy to test the formation of large complexes is size exclusion 3 chromatography (SEC), in which large complexes (in our case, fibrils) are excluded from the

4 separation column. To track Grh1 as it moves through the column, we employed the GFP-tagged 5 yeast strain. Yeast cells were cultivated under the desired condition: control, starvation, or

6 submitted to a temperature increase. The whole extract obtained from disrupted yeast cells in 7 each condition was then applied in a SuperDex200, and the proteins were tracked via their optical 8 absorbance at 280 and $395 \mathrm{~nm}$ for the target proteins and GFP, respectively. Figure 4A shows the 9 results of the SEC experiments. For clarity, elution profiles were normalized, and only the signals 10 at $395 \mathrm{~nm}$ are shown. In the control experiment (black line), the signal from GFP appeared 11 around $16 \mathrm{~mL}$ of elution. In starved cells (red line), the curve was shifted to the left, indicating 12 that GFP (and consequently Grh1) was then too large to enter the column, being excluded at 9 $13 \mathrm{~mL}$. On the other hand, there were two populations in the non-permissive temperature condition $14\left(37^{\circ} \mathrm{C}\right.$, blue line): one that was excluded from the column, and another one that left the column at the same point as in the control condition. While SEC does not give information about the type of structure formed, it does indicate that, under stress conditions, Grh1 undergoes changes that 17 significantly affect its size.

To explore whether the observed size change in Grh1 was due to protein aggregation, ultracentrifugation in SDS was used to differentiate amyloid fibrils from other possible amorphous aggregates of Grh1. ${ }^{[5]}$ We also used the Grh1-GFP strain and performed a dot blot (Figure 4B) to detect the presence of Grh1 in the non-solubilized pellet. Confirming the SEC

22 observations, Grh1-GFP fibrils were present under stress conditions but not in the control,

23 indicating that Grh1 formed SDS-insensitive large complexes when the yeast cells were 24 submitted to starvation or non-permissive temperature. Therefore, we can infer that the size 25 increase was not due to amorphous aggregation but rather to amyloid formation. 


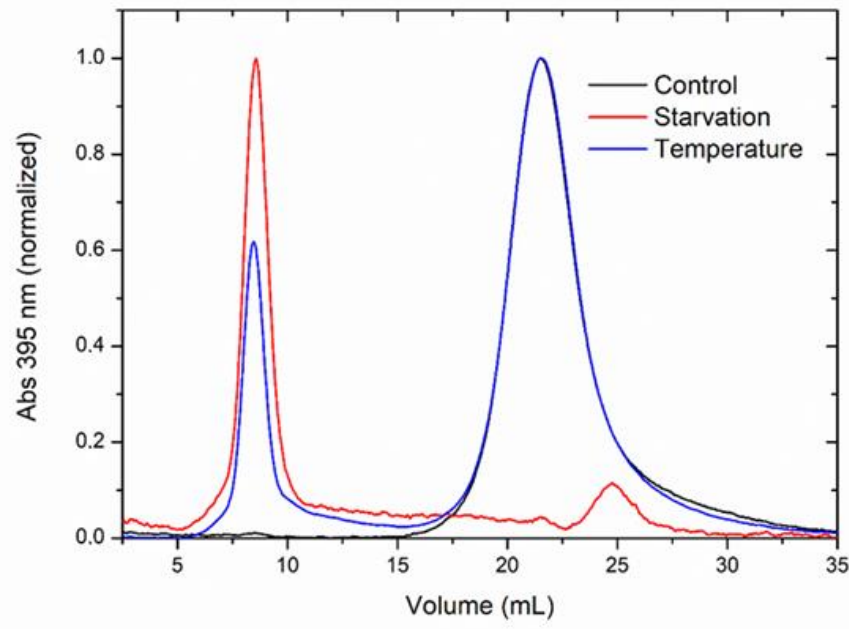

(A)

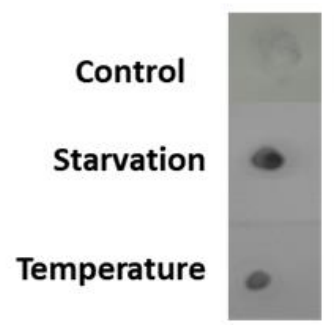

(B)

2 Figure 4: Results of the experiments monitoring the formation of higher-order complexes by 3 using the yeast strain producing a GFP-tagged Grh1. (A) Elution profiles (normalized) of the size 4 exclusion chromatography following the GFP signal (at $395 \mathrm{~nm}$ ). (B) Dot blot of the pellet 5 obtained from the ultracentrifuged samples. The detection was based on the use of an anti-GFP 6 antibody.

After incubation in starvation conditions, yeasts can return to their normal state when physiological conditions are restored. ${ }^{[25]}$ In agreement with that, our data show that there was no

11 autofluorescence from the sample that was subjected to starvation for 2 hours and then brought

12 back to normal conditions (Figure S4A). This suggests that all the fibrillation events indicated by

13 the autofluorescence in starvation conditions were reversible. Moreover, it is known that HSR

14 can sustain the stress for 2 hours and the cells can also go back to normal once the optimal 15 temperature is restored. ${ }^{[30]}$ Akin to starved cells, the autofluorescence from cells subjected to 16 heating at $37^{\circ} \mathrm{C}$ for 2 hours (heat shock) was no longer seen when the cells were brought back to $17 \quad 30^{\circ} \mathrm{C}$ (Figure S4B). 
Transmission Electron Microscopy (TEM) was used to visualize the assemblies formed by Grh1 in different conditions: purified Grh1 heated to $37^{\circ} \mathrm{C}$ for 30 minutes before the

3 preparation of the TEM grids; Grh1-GFP immunoprecipitated from control (optimal growth

4 conditions) cells and cells subjected to heat shock or starvation; pellets of ultracentrifuged WT

5 control cells and cells subjected to starvation. The results of each experiment are shown in Figure 65.

Samples of purified Grh1 resulted in the images of fibrils shown in the left panel of Figure 8 5A. Their amyloid signature has been previously confirmed. ${ }^{[21]}$ Curiously, besides the usual 9 fibrillar arrangement, it is also possible to observe the formation of a seemingly closed square10 like structure (red circle in the left panel of Figure 5A). The dimensions of the particles in the 11 grids (both the ones forming the square-like structure and the loose ones) were obtained by using 12 the software ImageJ (see yellow double arrows in the right panel of Figure 5A), yielding lengths 13 between 15 and $20 \mathrm{~nm}$, and thickness in the range of 4.0 to $7.5 \mathrm{~nm}$.

In Figure 5B, we have images of immunoprecipitated Grh1-GFP from cells in heat shock. In this case, the structures present in the grid looked more fibrillar than the ones from the in vitro experiment (purified Grh1, Figure 5A). Such fibrils seem to be formed by individual units that are linked together to build the complete structure. One of the individual units is marked with a red arrow in the left panel of Figure 5B. The size and thickness of those units are compatible with what was found for purified Grh1 (16 to $21 \mathrm{~nm}$ in length and 4.0 to $7.5 \mathrm{~nm}$ in thickness). It is possible again to find arrangements of the fibrils consisting of square-like structures (zoomed-in in the right panel of Figure 5B). For the sample immunoprecipitated from cells subjected to starvation, we obtained the images in Figure 5C. Akin to the heat-shock condition, we have fibrillike assemblies. It is also possible to identify small individual units (marked with red arrows in the left panel of Figure 5C). These have sizes and thickness similar to those found in the heatshock condition's grids (Figure 5B). Unlike the previous conditions, starved cells also gave rise to a structurally different arrangement (shown in the right panel of Figure 5C) obtained from another grid of the same experiment. In this case, even though it was difficult to differentiate all

28 the individual units that make up the whole structure, some of them could be distinguished (see red arrows), forming a more complex structural arrangement than those seen in Figures 5A-B. 
1 Such arrangement resembled a network of branched convoluted tubules. The dimensions of the

2 individual units were in the same range as before (16-22 $\mathrm{nm}$ of length and 4.5-5.8 $\mathrm{nm}$ of

3 thickness), and the average diameter of the larger structure was ca. $153 \mathrm{~nm}$.

$4 \quad$ Considering we know from previous experiments (Figure 4B) that Grh1 was found in the 5 pellet of starved cells subjected to ultracentrifugation, we further investigated the fibrillation in

6 starvation by taking the pellet of starved WT cells to the microscope and obtained images like the

7 ones in Figure 5D. After the ultracentrifugation, the type of arrangement found was slightly

8 different from those seen in the sample obtained using the immunoprecipitation protocol (Figure

9 5C). That can be due to the physical process of ultracentrifugation or to changes caused by GFP

10 in the structure. Nevertheless, one can again see small units forming interconnected fibril- or

11 square-like structures (left panel in Figure 5D). These squares can be seen in more detail in the

12 right panel of Figure 5D (marked with a blue square).

13 Interestingly, the dimensions of the squared structures (i.e., the size of their sides) were 14 around $21 \mathrm{~nm}$ of side length and $5 \mathrm{~nm}$ of thickness. These values are compatible with the ones

15 found in the experiments with purified Grh1 (in vitro) and immunoprecipitated samples in both 16 conditions (heat shock and starvation, in vivo). The ability to form somewhat short fibrils that can

17 apparently result in square-like structures seems to be the organization pattern adopted by Grh1.

Finally, it is worth mentioning that the grids containing Grh1-GFP immunoprecipitated

19 from cells grown in optimal growth conditions or pellet of ultracentrifuged cells grown in optimal 20 growth conditions neither showed any of the structures described above nor any other of 21 relevance. 


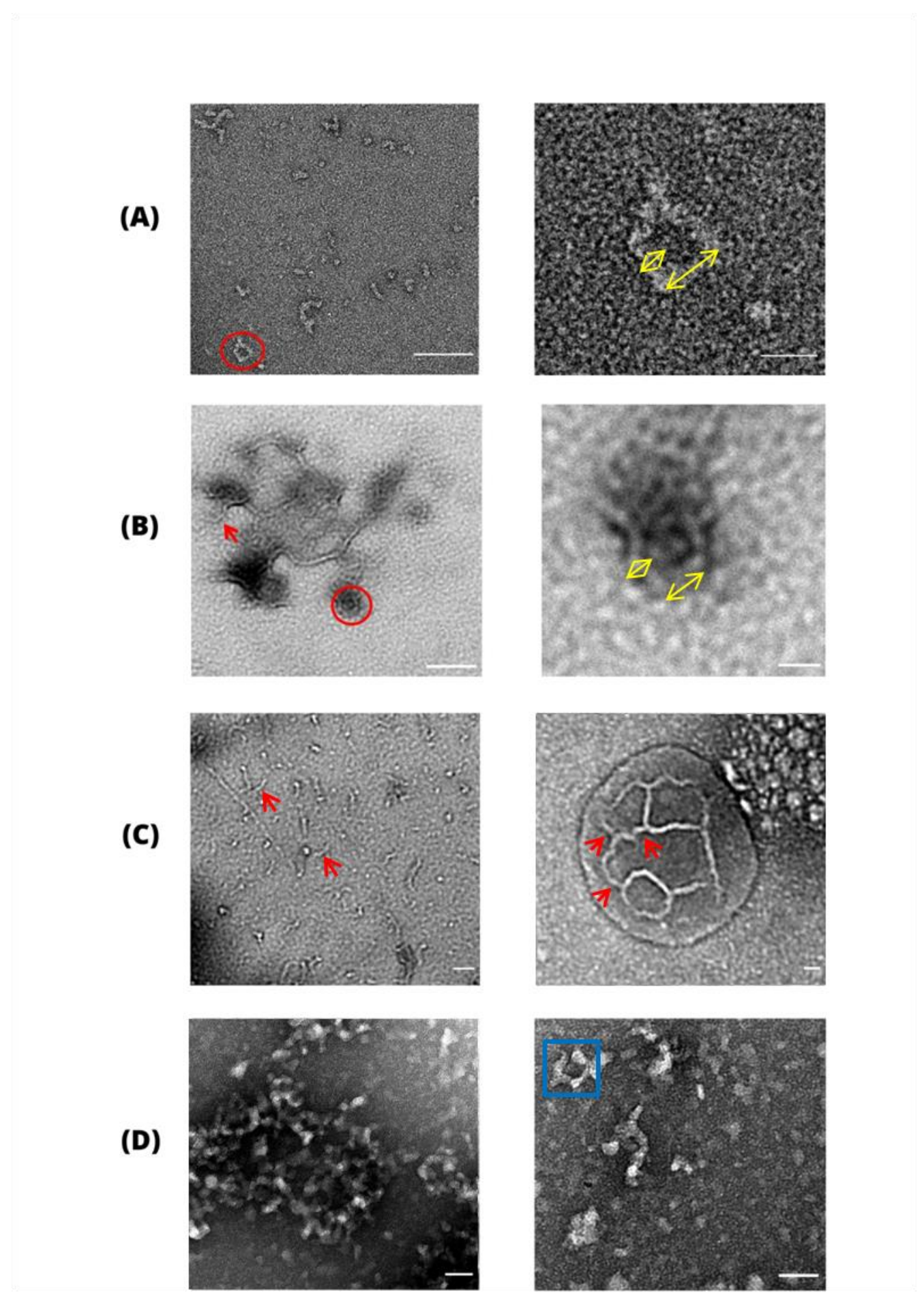

2 Figure 5: TEM images of: (A) purified Grh1 heated at $37^{\circ} \mathrm{C}$. (B) Grh1-GFP immunoprecipitated 3 from yeast cells in heat shock. Right panels are zoomed-in images of the red circle seen on the 4 left panels. Yellow double arrows indicate measured dimensions of particles. (C) Grh1-GFP 5 immunoprecipitated from yeast cells in starvation. Red arrows indicate units of fibrils. (D) Pellets 
1 of ultracentrifuged yeast cells in starvation. Blue square highlights the structures found on the

2 grids. Scale bar: (A), (B), (C) left panels:100 nm. (A), (B), (C) right panels, (D): $20 \mathrm{~nm}$.

3

4

\section{Discussion}

Amyloid formation inside cells has been a subject of significant interest over the years. It has gained even more attention lately since various aggregation-prone proteins, whose potential formation of amyloid-like structures is not disease-related, have been described. ${ }^{[2]}$ Nevertheless, detecting amyloid-like fibrils in vivo remains a challenge. Several reports have successfully probed aggregation within cells ${ }^{[5]}$ by using chimeras of the target protein tagged with fluorescent reporters. In the case of aggregation-prone proteins, the foci formed inside the cells can, in principle, be visualized under the fluorescence microscope. ${ }^{[5]}$ Despite the usefulness of this latter approach, in the specific case of Grh1, compartmentalization of the protein either in CUPS (in the case of starvation) or likely in stress granules during HSR can lead to the formation of 1 to 3 punctate structures, therefore hampering the direct identification of potential fibrils.

Grh1 fibrillation has been demonstrated in vitro ${ }^{[21]}$ and we further expanded the list of triggering factors of that process to now include acidic environments (Figure S1). However, its occurrence within the cell and its potential implications were still unclear. Fibrils and changes in the cytoplasmic state are seemingly tools used by the cell to cope with different types of stress. ${ }^{[2,}$ ${ }^{30]}$ Our initial hypothesis was then that fibrils of Grh1 could form in vivo and would be necessary under specific conditions. The test of our idea was mainly based on the use of a label-free assay, firstly described by Pinotsi et al. in 2013. ${ }^{[8]}$ Such a method for detecting amyloid fibrils relies on the intrinsic fluorescence in the UV-visible region that arises when the protein changes conformation and adopts the characteristic $\beta$-sheet rich structure of amyloids. Chan et al. [9] further described this fluorescence signature and how unlikely it would be for an experiment based solely on fibril autofluorescence to be used in vivo due to the competing autofluorescence from the cells. It was not a surprise, then, those publications using FLIM and intrinsic fluorescence that followed were based on either FRET experiments or changes in a reporter's physicochemical parameter when fibrillation happened. ${ }^{[10,11]}$ 
Therefore, although in vivo FLIM of proteins without a reporter may not always be possible, we could show this can be a valuable approach for yeast cells. We specifically tested

3 Grh1 in vivo fibrillation due to starvation (Type III trigger - Figures $2 \mathrm{~B}$ and $3 \mathrm{~B}$ ) or to

4 temperature increase (HSR trigger - Figure 2A), two stress scenarios where Grh1 seems to play a

5 central role. During starvation, Grh1 leaves the Golgi membrane and relocalizes to be part of

6 CUPS. ${ }^{[16]}$ Upon heat shock, although there is hitherto no study describing the direct participation

7 of Grh1, its increased expression when the yeast is subjected to thermal stress has been

8 reported. ${ }^{[31]}$ In control conditions, the autofluorescence signal arising from the cells was

9 negligible when excited at $375 \mathrm{~nm}$ (Figure 2C).

Our data showed that the autofluorescence could be used to monitor the formation of the

11 fibrils themselves (presence or absence - Figures 1, 2, and 3) and differences in the environment

12 surrounding them induced by the distinct stress sources (Figure 2D). More specifically, the

13 alterations in $\mathrm{pH}$, temperature, and starvation yielded distinguishable lifetime decays, therefore

14 strongly suggesting that the fibrils are in different environments, which agrees with the idea of

15 distinct cell responses and the corresponding Grh1 function in each case. It is interesting to note

16 that the temperature stress in both cell types ( $E$. coli and yeast) led to identical time decays,

17 which indicates that the cell's HSR involves the formation of the fibrils in somewhat similar environments.

To further infer the participation of Grh1 fibrillation in each stress condition, we obtained FLIM and time decay data from a Grh1-knockout yeast strain under heat shock and starvation (Figure 3). The images from the heat-shocked knockout cells, unlike the images of the heat-

22 shocked Grh1-containing cells (Figure 2C), surprisingly showed the cells as black dots, which indicates the lack of Grh1 led the cells to a different type of response to HS. As previously said, currently, no studies link Grh1 to the HSR. Still, some of the data available and our results

25 reinforce the hypotheses of fibrillation of Grh1 in the context of HS. Gasch et al., ${ }^{[31]}$ through microarray DNA experiments, measured the changes in transcript levels over time in response to several types of stress, including temperature. For Grh1, what they observed was an increase in 28 transcription when the cell was subjected to heat shock $\left(37^{\circ} \mathrm{C}\right) .{ }^{[31]}$ Considering a stress situation where the cell stops its non-essential activities to save as much energy as possible, an increase in 
1 Grh1 transcription suggests a protein function in that scenario. Besides that, when the Grh1-GFP

2 cell is subjected to a non-permissive temperature $\left(37^{\circ} \mathrm{C}\right)$, the signal from GFP, which was

3 initially more dispersed, coalesced into foci inside the cell, thus becoming brighter spots than

4 before (Figure S5). As described by Alberti, Halfmann, and Lindquist, ${ }^{[5]}$ proteins that fibrillate in

5 vivo coalesce into microscopic assemblies, just like the ones we observed for Grh1.

On the other hand, the knockout cells showed a distinct response to starvation compared to HS. In this case, FLIM signals could be detected (Figure 3B), thus suggesting fibrillation of 8 other proteins in the cell, described before for the protein Cdc19. ${ }^{[34]}$ Despite the existence of this non-Grh1-related signal, we could find differences in the time decays (Figure 3C) and their distributions (Figure S2) measured from WT and knockout cells. Furthermore, sedimentation

11 experiments and a modified version of the filter retardation assay (in the form of an SEC experiment $)^{[5]}$ were used to corroborate our FLIM data by showing that Grh1 was present in the fibrils formed within the yeast cells. To do so, we used a yeast strain expressing Grh1 tagged with GFP to allow for the immunoprecipitation of the Grh1-GFP chimera. The fibrils of Grh1GFP were then detected using FLIM (Figure S3B) and compared with the data obtained for the whole yeast cell (Figure 2B). The pattern observed in those images and the similar lifetimes measured in both experiments (Figure S3C) allowed us to infer that the in-cell fibrillation was indeed due to Grh1.

To enhance our understanding of the fibril morphology, we used transmission electron microscopy to visualize the structures formed by Grh1. The TEM images from purified Grh1, immunoprecipitated Grh1-GFP from cells in heat shock and starvation, and ultracentrifuged WT cells in starvation (Figure 5) all showed similar patterns where one can see the coexistence of fibril-like and square-like structures. The experiments here reported did not allow for atomicresolution structural determination of Grh1-containing assemblies. Nevertheless, it was possible to distinguish short linear units in some images, whose dimensions are ca. $20 \mathrm{~nm}$ long and $6 \mathrm{~nm}$ thick. They are apparently linked to form the fibril itself and other more complex arrangements. Bruns et al. ${ }^{[32]}$ showed that, upon starvation, Grh1 concentrates in large membraneous punctae in 28 the cell to form the so-called compartment for unconventional protein secretion (CUPS). Despite the thorough description of the biogenesis of this new compartment, its detailed structure and 
1 composition were still not clear. Curwin et al. ${ }^{[33]}$, in an elegant combination of correlative light

2 and electron microscopy (CLEM) and fluorescence, advanced the knowledge on that issue by

3 reporting CUPS were arranged in a somewhat spherical structure of convoluted tubules and

4 vesicles, whose average diameter was ca. $200 \mathrm{~nm}$. In the same paper, the authors proposed a

5 pathway for CUPS formation that would consist of the segregation of Grh1 in tubular clusters,

6 followed by the engulfment of this immature CUPS by a sheet-like structure called saccule. The

7 exact origin of the saccule membrane was not determined, but Grh1-containing membranes were

8 suggested as one possibility. Our TEM data (Figure 5) offer another possibility: the Grh1 higher-

9 order structural arrangements. The GRASP's ability to form fibrillar structures, Grh1 among

10 them, has been demonstrated. ${ }^{[21]}$ In the Grh1 case, combining individual somewhat linear units

11 seems to give rise to distinct 3D structures, such as the fibrils and the square-like seen in Figures

$125 \mathrm{~B}-\mathrm{C}$. Furthermore, in the right panel of Figure 5C, an even more complex arrangement

13 involving an apparent network of tubules, whose rough average diameter was around $150 \mathrm{~nm}$,

14 can be seen. The dimensions of the chains formed by the units reported here are compatible with

15 the images showing the different stages of CUPS maturation described by Curwin et al. ${ }^{[33]}$ as the

16 saccule. We could speculate that the 3D-sheet of unknown origin engulfing the immature CUPS

17 and even the Grh1-containing vesicles described by Curwin et al. could be formed by Grh1

18 higher-order structures rather than their monomers. The reversibility of Grh1 fibril formation

19 (Figure S4) is also compatible with a mechanism that needs to be turned on and off depending on

20 the conditions triggering the cell stress.

21 Our data do not rule out the participation of secretory and endosomal membranes, but

22 Grh1 structural plasticity and its ability to form complex arrangements seem to be a new piece of

23 information that needs to be added as an alternative in the formation of compartments for UPS as

24 well as for heat shock response. Furthermore, the capacity of Golgi-related proteins to undergo

25 liquid-liquid phase separation (LLPS) has been recently demonstrated ${ }^{[34]}$, which raises another

26 exciting possibility regarding whether GRASP could also undergo such transition, a feature yet to

27 be determined. LLPS as the source of membrane-less compartments has gained particular interest

28 over the last few years ${ }^{[35]}$, and it might well be another aspect to consider when tackling the

29 problem of UPS and HSR. 
In summary, here, we present solid evidence of in vivo formation of Grh1 fibrils under stress conditions. While the details on how exactly they function in both Type III UPS and HSR

3 are yet to be fully revealed, our data reinforce the new concept of functional fibrils in yeasts as

4 active factors in response to certain types of stress. As any first-time idea appearing in the

5 literature, our findings seem to bring more questions than answers. Still, they undoubtedly offer

6 new perspectives to better understand amyloids in vivo and Grh1 roles in UPS and HSR.

\section{Materials and Methods}

\section{Protein Expression and Purification}

The protocol for Grh1 expression in E. coli and purification is described elsewhere. ${ }^{[21]}$ For the experiments with $E$. coli in acidic $\mathrm{pH}$, cells were grown, and expression was induced and carried out for 18 hours at $20^{\circ} \mathrm{C}$. Cells were pelleted by centrifugation at 8,000g for 10 minutes, transferred for LB medium added of $50 \mathrm{mM}$ MES and $20 \mathrm{mM}$ sodium acetate, $\mathrm{pH}$ 4.6, and kept shaking at $20^{\circ} \mathrm{C}$ for 1 hour.

\section{Yeast Culture}

The Saccharomyces cerevisiae strain Y470 was used in the FLIM experiments with excitation at $375 \mathrm{~nm}$. All the other experiments without tagging or knockout were performed in the parental strain BY4741. The GFP-tagged and the knockout strains come from the commercial Yeast GFP clone collection (ThermoFisher Scientific). All cultures were grown in YPD medium (1\% Yeast Extract, 2\% Peptone, 2\% Glucose).

For all experiments, a colony of the desired strain was placed in liquid YPD or SC-ura and allowed to grow overnight at $30^{\circ} \mathrm{C}$. The following morning the colony was transferred to 200 $\mathrm{ml}$ of YPD and allowed to grow again, this time under slow agitation until the culture reached an optical density at $600 \mathrm{~nm}$ of ca. 0.5 .

For starvation, cultures were centrifuged and resuspended in $2 \%$ potassium acetate solution (as described by Cruz-Garcia et al. ${ }^{[25]}$ ). For HSR, cultures were placed under agitation at 
$137^{\circ} \mathrm{C}$. Both experiments were run for $2 \frac{1}{2}$ hours, and the same time was used for recovery, where

2 the cultures were put back at optimal growth conditions.

\section{Immunoprecipitation}

Immunoprecipitation was carried out using the GFP-tagged strain with the GFP-Trap

5 Magnetic Agarose (Chromotek). Cells in the desired conditions were centrifuged at 12,000g for

610 minutes, and the supernatant was collected. The beads were previously equilibrated with

7 dilution/washing buffer (10 mM Tris pH 7.5, $150 \mathrm{mM} \mathrm{NaCl}, 0.5 \mathrm{mM}$ EDTA. $\mathrm{pH}$ adjusted to 8).

8 Beads were added to the supernatant, and the solution was left rotating end-to-end for 1 hour at

$9 \quad 4^{\circ} \mathrm{C}$. Two washing steps were performed by separating the beads with a magnet until the

10 supernatant was clear, discarding the supernatant, and resuspending beads with washing buffer.

For transmission electron microscopy experiments, the proteins were eluted by adding

$1250 \mu \mathrm{L} 0.2 \mathrm{M}$ glycine $\mathrm{pH}$ 5.5. Although the protocol recommends $\mathrm{pH} 2.5$, given that Grh1

13 fibrillates in $\mathrm{pH}$ below 5.5, we decided to use this value (5.5) to avoid undesired fibrillation in the

14 samples. The samples were incubated for 30 seconds under constant agitation, the supernatant

15 was placed in another Eppendorf tube, and the solution neutralized with $5 \mu \mathrm{L} 1 \mathrm{M}$ Tris base, $\mathrm{pH}$

16 10.4. For confocal and FLIM experiments, since the presence of the beads was not a problem, the

17 elution step was not performed, and the beads with trapped GFP were taken to the microscope.

\section{Fluorescence Lifetime Imaging Microscopy}

19 FLIM experiments were performed in an IX71 Inverted Microscope (Olympus) equipped with a

20 PicoQuant MT 200 confocal module. Excitation was set at $375 \mathrm{~nm}$, and emission was detected

21 from $405 \mathrm{~nm}$. Data collection and analysis were performed with the SymPhoTime 64 software

22 (PicoQuant). A Region of Interest (ROI) was delimited for analysis, and the nExponential Tailfit

23 mode was selected. The number of exponential components was chosen based on the best

24 distribution of the residuals and the best $\mathrm{x}^{2}$ value found. To obtain the lifetime's histograms, the

25 FLIM fit function was used based on the manual provided by PicoQuant (available at:

26 https://www.picoquant.com/images/uploads/page/files/17319/4_td_flim.pdf). Decay curves

27 presented are the best fits for each of the results. 


\section{Multiphoton Microscopy}

Images were acquired using a LSM 780 Multiphoton AxioObserver (Zeiss), equipped with a titanium sapphire laser. Excitation was set at $880 \mathrm{~nm}$, and emission recorded with a 515/30

$4 \mathrm{~nm}$ filter. The microscope was available at the Laboratório Multiusuário de Microscopia

5 Multifóton (Departamento de Biologia Celular e Molecular e Bioagentes Patogênicos, Faculdade

6 de Medicina de Ribeirão Preto, Universidade de São Paulo).

\section{Gel Filtration}

Cultures in different conditions were centrifuged and pelleted in PBS, followed by sonication for 7 minutes, in cycles of $30 \mathrm{sec}$ on and $30 \mathrm{sec}$ off. To separate solid particulates, 10 cultures were centrifuged at $20,000 \mathrm{~g}$ for 20 minutes, and the collected supernatant was 11 concentrated using a Vivaspin column (GE Healthcare, Buckinghamshire, United Kingdom). The 12 concentrated supernatant was applied to a Superdex200 10/300 GL gel filtration column (GE 13 Healthcare, Buckinghamshire, United Kingdom).

\section{Ultracentrifugation}

$2 \mathrm{~mL}$ of cultured cells were harvested and resuspended in $300 \mathrm{uL}$ of lysis buffer $(50 \mathrm{mM}$ 16 Tris, pH 7.5, $150 \mathrm{mM} \mathrm{NaCl}, 2 \mathrm{mM}$ EDTA, 1mM PMSF, 5\% glycerol). Disruption was carried out by vortexing with acid-washed glass beads. $300 \mu \mathrm{L}$ of RIPA buffer (50 mM Tris, pH 7.0, 150 $\mathrm{mM} \mathrm{NaCl}, 1 \%$ Triton $\mathrm{X}-100,0.1 \%$ SDS) were added to the solution, which was vortexed again

19 for $10 \mathrm{sec}$. Centrifugation at $1,500 \mathrm{~g}$ for 5 minutes was used to pellet cell debris. $200 \mu \mathrm{L}$ of the 20 soluble fraction was centrifuged in a TLA 100-2 rotor for $45 \mathrm{~min}$ at $100,000 \mathrm{~g}$ and $4{ }^{\circ} \mathrm{C}$ in an 21 Optima TL Beckman ultracentrifuge.

\section{Dot Blot}

$10 \mu \mathrm{L}$ of resuspended precipitate (see Ultracentrifugation) was blotted in nitrocellulose membrane, which was then blocked with Blocking buffer (TBST - $10 \mathrm{mM}$ Tris, $150 \mathrm{mM} \mathrm{NaCl}$,

$250.1 \%$ Tween 20 - plus $1 \%$ w/v BSA) for 24 hours. Incubation with rabbit-polyclonal anti-GFP primary antibody in a 1:10,000 dilution (Invitrogen Cat. Number \#A-11122) in Blocking Buffer was carried out for one hour, followed by three washes with TBST. Incubation with anti-Rabbit 
1 Secondary Antibody Solution Alk-Phos. Conjugated (Invitrogen ${ }^{\mathrm{TM}}$ ) followed and preceded for

2 one hour. Washing and detection were performed as recommended for Novex ${ }^{\circledR}$ AP Chromogenic

3 Substrate.

The purified protein was analyzed at the Brazilian Nanotechnology National Laboratory in a JEOL 3010. The ultracentrifuged and immunoprecipitated samples were analyzed in an FEI

8 Tecnai 12 Transmission Electron Microscope at the Sir William Dunn School of Pathology of the

9 University of Oxford (UK). A $120 \mathrm{kV}$ of acceleration voltage was applied on the samples

10 deposited in a $15 \mathrm{~mA}$ discharged copper grid and stained with $2 \%$ uranyl acetate. The images

11 were analyzed with ImageJ. ${ }^{[36]}$

Author Contributions: NAF performed most of the experimental procedures and analyses. ADR was involved in part of the experimental procedures. AW and PSRC coordinated part of the project. AJCF conceived and coordinated the project. All authors also approved the final version of the manuscript.

Competing Interest Statement: The authors declare no competing interests.

\section{Acknowledgments}

The authors acknowledge Fundação de Amparo à Pesquisa do Estado de São Paulo (FAPESP) for the financial support (Grant no. 2015/50366-7, 2012/20367-3 and 2008/55831-6). NAF and ADR thank FAPESP for the Ph.D. and undergraduate fellowships (Grant no. 2016/23863-2 and 2018/24968-8). AJCF thanks Conselho Nacional de Desenvolvimento Científico e Tecnlógico (CNPq) for the partial financial support (Grant no. 306682/2018-4). The authors also thank Prof. Cleslei Fernando Zanelli (Department of Biological Sciences, School of Pharmacy, UNESP, Brazil) for the Grh1 GFP-tagged and Grh1 knockout yeast strains. 


\section{References}

[1] E. H. Koo, P. T. Lansbury, J. W. Kelly, Proceedings of the National Academy of Sciences of the United States of America 1999, 96 (18), 9989, https://doi.org/10.1073/pnas.96.18.9989.

[2] G. Cereghetti, S. Saad, R. Dechant, M. Peter, Cell Cycle 2018, 17 (13), 1545, https://doi.org/10.1080/15384101.2018.1480220.

[3] M. G. Iadanza, M. P. Jackson, E. W. Hewitt, N. A. Ranson, S. E. Radford, Nature Reviews Molecular Cell Biology 2018, 19 (12), 755, https://doi.org/10.1038/s41580-018-0060-8.

[4] Z. L. Almeida, R. M. M. Brito, Molecules 2020, 25 (5), https://doi.org/10.3390/molecules25051195.

[5] S. Alberti, R. Halfmann, S. Lindquist, Methods in Enzymology, Vol 470: Guide to Yeast Genetics:: Functional Genomics, Proteomics, and Other Systems Analysis, 2nd Edition 2010, 470, 709, https://doi.org/10.1016/s0076-6879(10)70030-6.

[6] M. T. Elghetany, A. Saleem, Stain Technology 1988, 63 (4), 201, https://doi.org/10.3109/10520298809107185.

[7] E. M. Reiman, W. J. Jagust, Neuroimage 2012, 61 (2), 505, https://doi.org/10.1016/i.neuroimage.2011.11.075.

[8] D. Pinotsi, A. K. Buell, C. M. Dobson, G. S. K. Schierle, C. F. Kaminski, Chembiochem 2013, 14 (7), 846, https://doi.org/10.1002/cbic.201300103.

[9] F. T. S. Chan, G. S. K. Schierle, J. R. Kumita, C. W. Bertoncini, C. M. Dobson, C. F. Kaminski, Analyst 2013, 138 (7), 2156, https://doi.org/10.1039/c3an36798c.

[10] E. K. Esbjorner, F. Chan, E. Rees, M. Erdelyi, L. M. Luheshi, C. W. Bertoncini, C. F. Kaminski, C. M. Dobson, G. S. K. Schierle, Chemistry \& Biology 2014, 21 (6), 732, https://doi.org/10.1016/i.chembiol.2014.03.014.

[11] G. S. K. Schierle, C. W. Bertoncini, F. T. S. Chan, A. T. van der Goot, S. Schwedler, J. Skepper, S. Schlachter, T. van Ham, A. Esposito, J. R. Kumita, E. A. A. Nollen, C. M. Dobson, C. F. Kaminski, Chemphyschem 2011, 12 (3), 673, https://doi.org/10.1002/cphc.201000996.

[12] F. A. Barr, M. Puype, J. Vandekerckhove, G. Warren, Cell 1997, 91 (2), 253, https://doi.org/10.1016/s0092-8674(00)80407-9.

[13] C. Viotti, in Unconventional Protein Secretion, Humana Press, New York 2016.

[14] C. Rabouille, V. Malhotra, W. Nickel, Journal of Cell Science 2012, 125 (22), 5251, https://doi.org/10.1242/jcs.103630.

[15] W. Nickel, C. Rabouille, Nature Reviews Molecular Cell Biology 2009, 10 (2), 148, https://doi.org/10.1038/nrm2617.

[16] F. Giuliani, A. Grieve, C. Rabouille, Current Opinion in Cell Biology 2011, 23 (4), 498, https://doi.org/10.1016/i.ceb.2011.04.005.

[17] D. Brough, P. Pelegrin, W. Nickel, Journal of Cell Science 2017, 130 (19), 3197, https://doi.org/10.1242/jcs.204206.

[18] V. Malhotra, Embo Journal 2013, 32 (12), 1660, https://doi.org/10.1038/emboj.2013.104.

[19] D. Cruz-Garcia, V. Malhotra, A. J. Curwin, Seminars in Cell \& Developmental Biology 2018, 83, 22, https://doi.org/10.1016/j.semcdb.2018.02.021.

[20] L. F. S. Mendes, A. F. Garcia, P. S. Kumagai, F. R. de Morais, F. A. Melo, L. Kmetzsch, M. H. Vainstein, M. L. Rodrigues, A. J. Costa, Scientific Reports 2016, 6, https://doi.org/10.1038/srep29976; L. F. S. Mendes, L. G. M. Basso, P. S. Kumagai, R. Fonseca-Maldonado, A. J. Costa, Biochimica Et Biophysica Acta-General Subjects 2018, 1862 (4), 855, https://doi.org/10.1016/j.bbagen.2018.01.009; L. F. S. Mendes, N. A. Fontana, C. G. Oliveira, M. Freire, J. L. S. Lopes, F. A. Melo, A. J. Costa-Filho, Febs Journal 
2019, 286 (17), 3340, https://doi.org/10.1111/febs.14869; S. T. Reddy, L. F. S. Mendes, N. A. Fontana, A. J. Costa, International Journal of Biological Macromolecules 2019, 135, 481, https://doi.org/10.1016/j.ijbiomac.2019.05.089; L. F. S. Mendes, M. R. B. Batista, P. J. Judge, A. Watts, C. Redfield, A. J. Costa, Febs Journal 2020, 287 (15), 3255, https://doi.org/10.1111/febs.15206.

[21] N. A. Fontana, R. Fonseca-Maldonado, L. F. S. Mendes, L. P. Meleiro, A. J. Costa, Scientific Reports 2018, 8, https://doi.org/10.1038/s41598-018-33955-1.

[22] S. T. Reddy, V. N. Uversky, A. J. Costa-Filho, Int J Biol Macromol 2020, 162, 1982, https://doi.org/10.1016/j.ijbiomac.2020.08.126.

[23] T. S. Reddy, V. N. Uversky, A. J. Costa-Filho, Eur Biophys J. 2020, 49(2), 133, https://doi.org/10.1007/s00249-019-01419-7.

[24] L. F. S. Mendes, N. A. Fontana, S. T. Reddy, V. N. Uversky, A. J. Costa-Filho, Int J Biol Macromol 2020, https://doi.org/10.1016/j.ijbiomac.2020.08.203.

[25] D. Cruz-Garcia, A. J. Curwin, J. F. Popoff, C. Bruns, J. M. Duran, V. Malhotra, Journal of Cell Biology 2014, 207 (6), 695, https://doi.org/10.1083/icb.201407119.

[26] S. Lindquist, Annual Review of Biochemistry 1986, 55, 1151, https://doi.org/10.1146/annurev.bi.55.070186.005443.

[27] R. Orij, J. Postmus, A. Ter Beek, S. Brul, G. J. Smits, Microbiology (Reading) 2009, 155 (Pt 1), 268, https://doi.org/10.1099/mic.0.022038-0.

[28] J. C. Wilks, J. L. Slonczewski, J Bacteriol 2007, 189 (15), 5601, https://doi.org/10.1128/JB.0061507.

[29] A. Jain, C. Blum, V. Subramaniam, in (Ed.: Elsevier), 2009, Ch. 4.

[30] S. Saad, G. Cereghetti, Y. Feng, P. Picotti, M. Peter, R. Dechant, Nat Cell Biol 2017, 19 (10), 1202, https://doi.org/10.1038/ncb3600; E. W. Wallace, J. L. Kear-Scott, E. V. Pilipenko, M. H. Schwartz, P. R. Laskowski, A. E. Rojek, C. D. Katanski, J. A. Riback, M. F. Dion, A. M. Franks, E. M. Airoldi, T. Pan, B. A. Budnik, D. A. Drummond, Cell 2015, 162 (6), 1286, https://doi.org/10.1016/i.cell.2015.08.041.

[31] A. P. Gasch, P. T. Spellman, C. M. Kao, O. Carmel-Harel, M. B. Eisen, G. Storz, D. Botstein, P. O. Brown, Mol Biol Cell 2000, 11 (12), 4241, https://doi.org/10.1091/mbc.11.12.4241.

[32] C. Bruns, J. M. McCaffery, A. J. Curwin, J. M. Duran, V. Malhotra, J Cell Biol 2011, 195 (6), 979, https://doi.org/10.1083/icb.201106098.

[33] A. J. Curwin, N. Brouwers, M. Alonso Y Adell, D. Teis, G. Turacchio, S. Parashuraman, P. Ronchi, V. Malhotra, Elife 2016, 5, https://doi.org/10.7554/eLife.16299.

[34] P. Ziltener, A. A. Rebane, M. Graham, A. M. Ernst, J. E. Rothman, FEBS Lett 2020, 594 (19), 3086, https://doi.org/10.1002/1873-3468.13884.

[35] S. Boeynaems, S. Alberti, N. L. Fawzi, T. Mittag, M. Polymenidou, F. Rousseau, J. Schymkowitz, J. Shorter, B. Wolozin, L. Van Den Bosch, P. Tompa, M. Fuxreiter, Trends Cell Biol 2018, 28 (6), 420, https://doi.org/10.1016/j.tcb.2018.02.004.

[36] C. T. Rueden, J. Schindelin, M. C. Hiner, B. E. DeZonia, A. E. Walter, E. T. Arena, K. W. Eliceiri, BMC Bioinformatics 2017, 18 (1), 529, https://doi.org/10.1186/s12859-017-1934-z. 